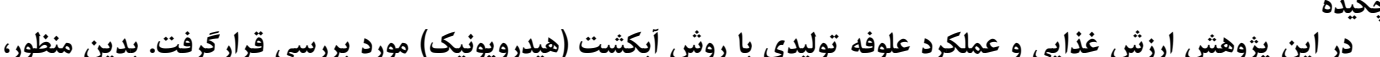

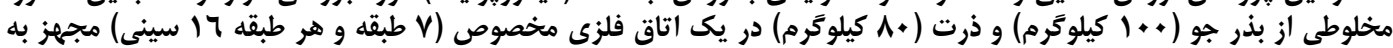

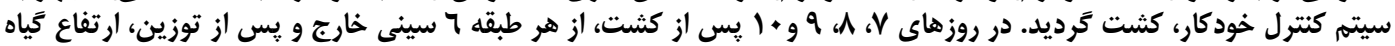

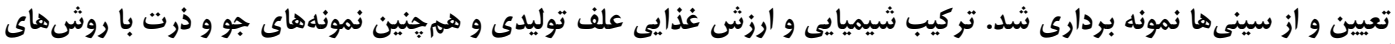

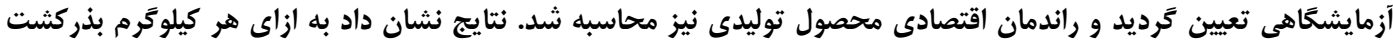

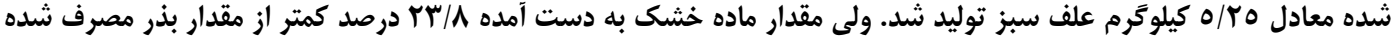

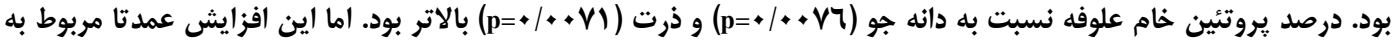

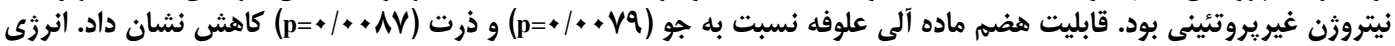

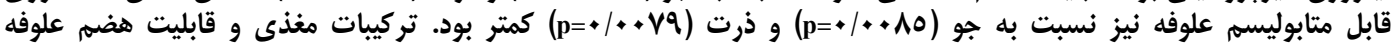

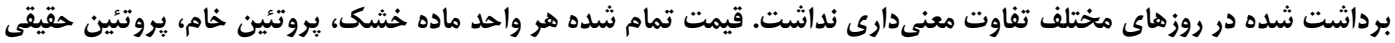

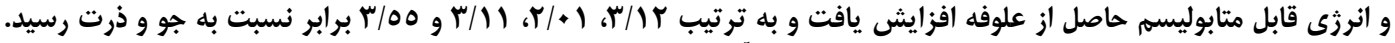

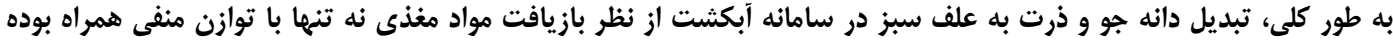

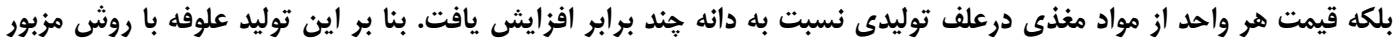

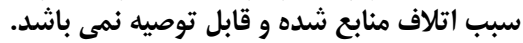

وازههاى كليدى: آبكشت، بازده، جو، ذرت، علوفه

مغذى در علوفه توليدى به دليل كاهش نسبى موادى مانند

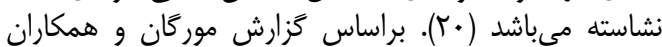

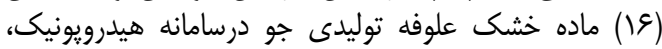

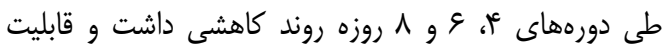

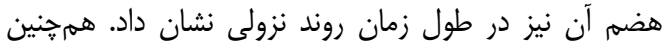

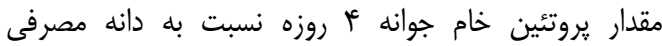

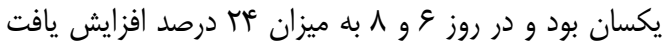

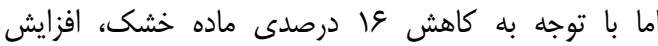

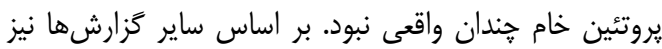

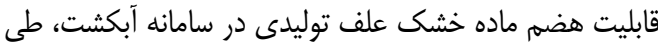

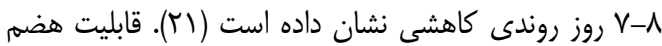

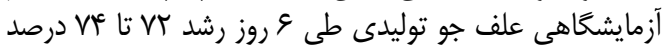

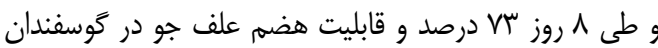

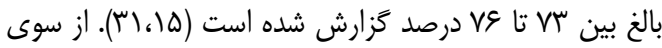

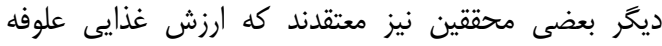

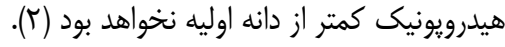

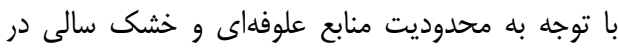

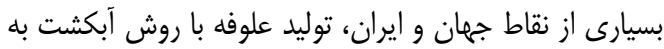

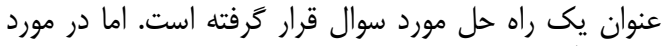

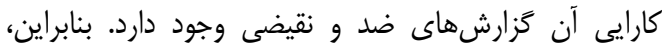

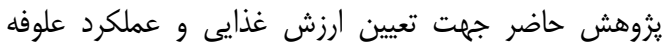

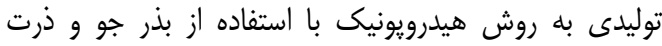

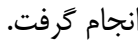

مقدمه

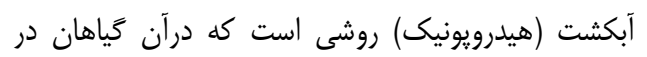

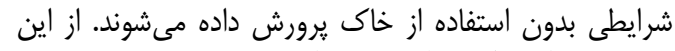

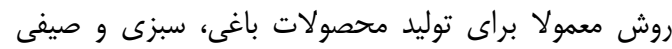

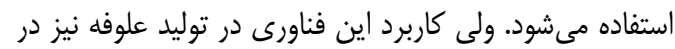

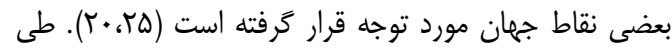

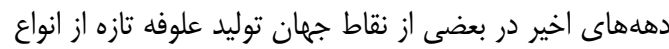

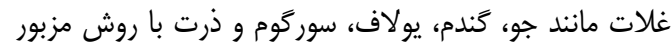

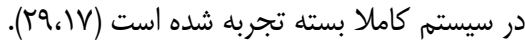

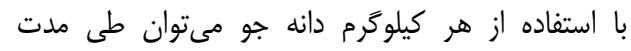

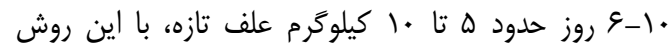

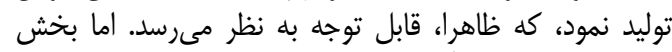

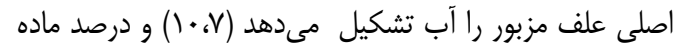

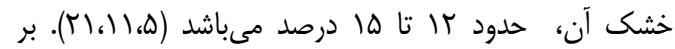

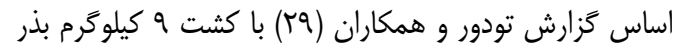

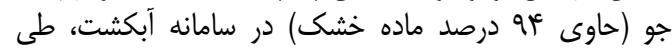

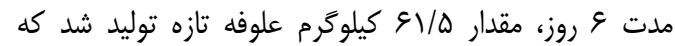

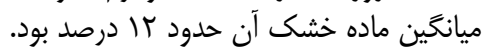

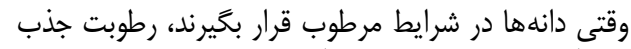

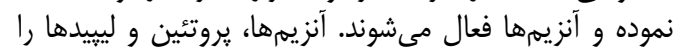

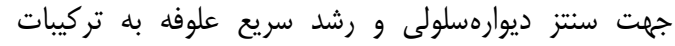

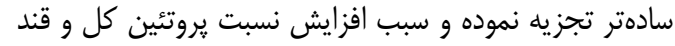

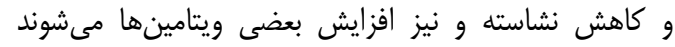

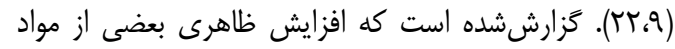


و ميزان جذب تعيين شد. آنغاه با استفاده از منحنسى استاندارد

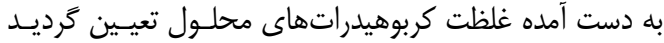

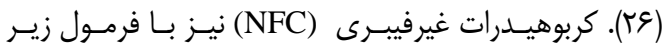
محاسبه شد (1): (1): (1)

$\mathrm{NFC}=1 \cdots-(\% \mathrm{NDF}+\% \mathrm{CP}+\% \mathrm{Ash}+\% \mathrm{Fat})$

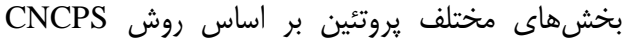

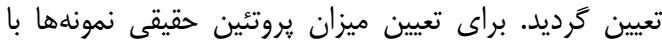

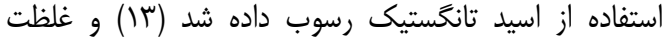

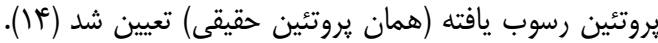

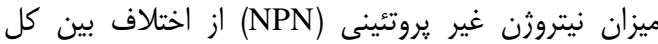

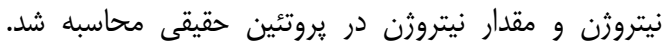

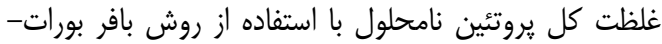

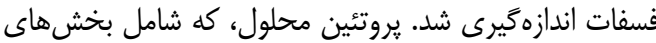
خ و B

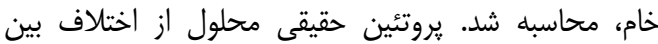

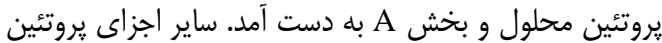

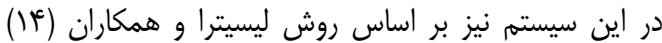

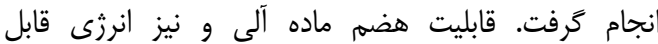

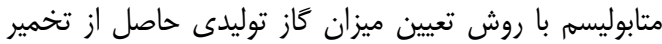

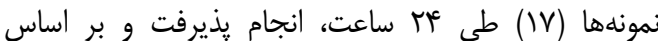
رابطههاى زير محاسبه شد (1) (1).

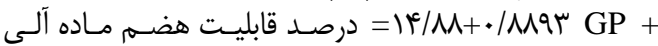
$.1+\operatorname{cr} \mathrm{CP}+.1 .901 \mathrm{XA}$

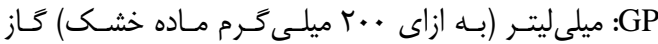

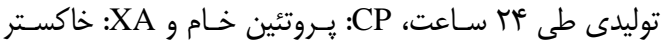

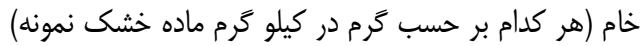
$\mathrm{ME}=r / r+\cdot / 1 \mathrm{r} \Delta \mathrm{V} \mathrm{GP}+\cdot / \cdot \Delta V \mathrm{CP}+\cdot 1 \cdot+r \Delta \wedge q$ $\mathrm{CP}^{r}$

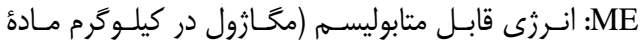

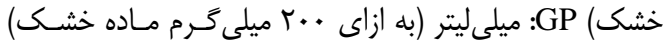

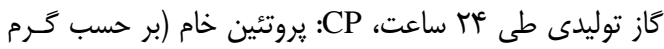

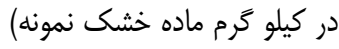
محاسبه بازده توليد

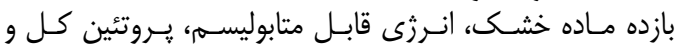

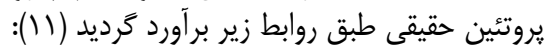

\section{مواد و روشها} توليد علوفه براى توليد علوفه علوفه از يك اتاق متر كشت فلزى در ابعاد ع

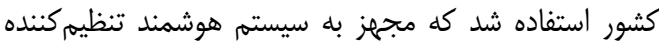

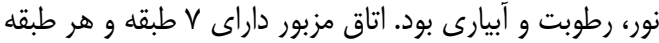

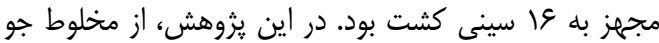

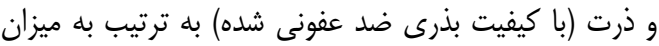

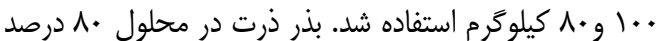

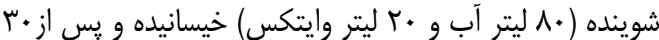

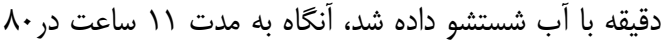

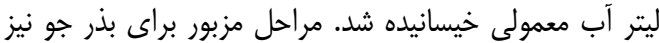

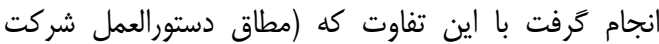

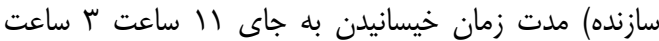

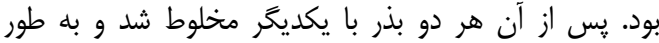

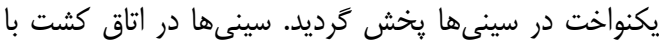
دماى ال M درجه سانتى

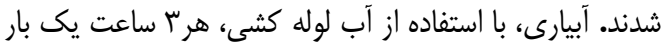

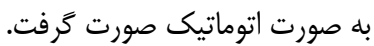

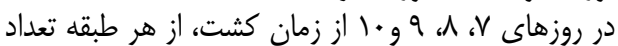

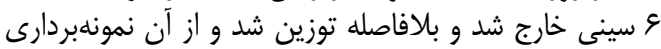

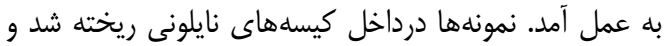

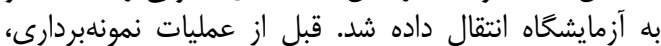

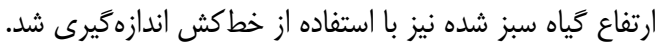

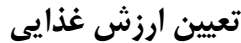

تركيبات شيميايى شامل مارئ ماده خشك، خاكستر خام، هربى

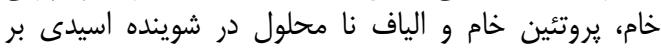

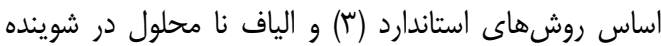

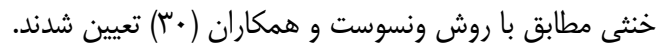

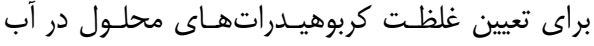

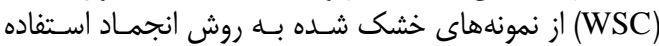

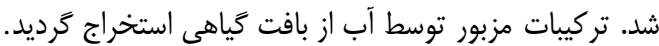

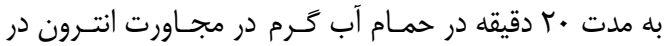

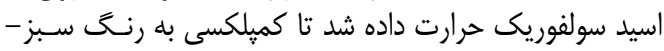

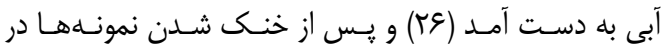

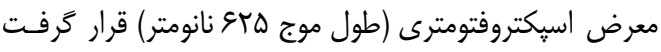

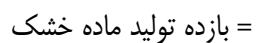

بازده توليد انرزى قابل متابوليسم =

بازده توليد يروتئين حقيقى =
وزن ماده خشك توليد شده

م مزن ماده خشك مصرف شده (بذر)

انرزى قابل متابوليسم حاصل از علوفه توليد شده

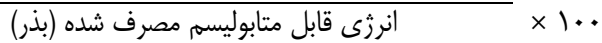

وزن يروتئين حقيقى توليد شده

وزن يروتئين حقيقى مصرف شيقي توليد (بذهر) 
عملكرد توليد علوفه

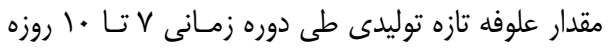

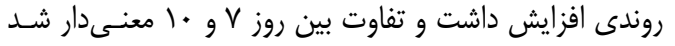

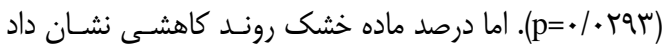

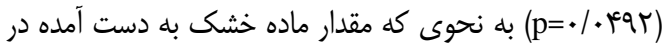

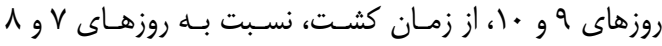

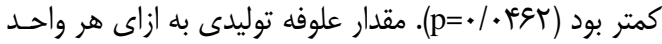

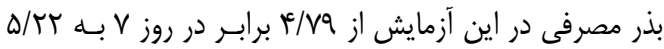

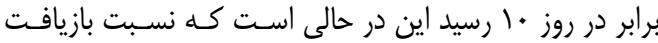

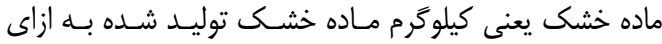

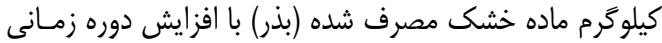

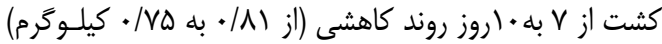

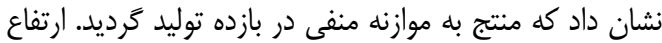

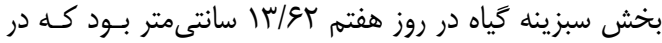

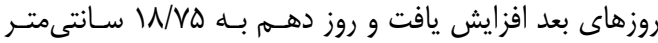

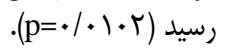

برآورد هزينه نسبى

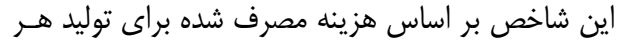

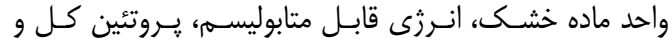

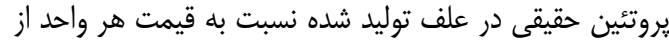
متغيرهاى ذكر شده دردانه جو و ذرت درت محاسبه شد.

تجزيه و تحليل آمارى دي داري

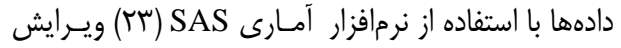

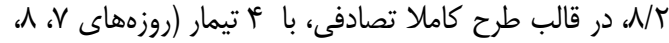

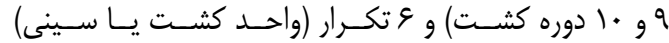

$\mathrm{Y}_{\mathrm{ij}}=\mu+\mathrm{T}_{\mathrm{i}+} \mathrm{e}_{\mathrm{ij}}$ بر اساس مدل زير مورد تجزيه آمارى قرار كَرفت:

در اين مدل، : مقدار هر مشاهده، : ميانكين صفت مورد مطالعل، مقايسه ميانكينها به روش آزمون جند دامنهائ دار دانكن انجام

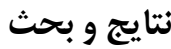

Table 1. Forage yield performance

\begin{tabular}{|c|c|c|c|c|c|c|}
\hline \multirow{2}{*}{ معنى احتمال } & \multirow{2}{*}{ اشتباه استاندارد } & \multicolumn{4}{|c|}{ زمان برداشت علوفه (روز) } & \multirow{2}{*}{ فراسنجهها } \\
\hline & & 1. & 9 & $\wedge$ & $\mathrm{V}$ & \\
\hline \multicolumn{7}{|c|}{ مقدار بذر مصرفى در هر سينى (كرم) } \\
\hline- & - & $\left|V^{\mu}\right|$ & $|V|$ & $\left|V^{2}\right|$ & 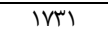 & بر حسب معمول \\
\hline- & - & $1 \Delta \Delta V$ & $1 Q \Delta V$ & $10 \Delta V$ & $I \Delta \Delta V$ & بر حسب ماده خشك \\
\hline \multicolumn{7}{|c|}{ علوفه توليدى در هر سينى } \\
\hline 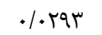 & $r q / v$. & $q \cdot r \Delta^{\mathrm{a}}$ & $1999^{\mathrm{a}}$ & $\Lambda \Gamma \Delta \cdot{ }^{\circ}$ & הזA & علف تازه (كَرم) \\
\hline.$/ . \mathrm{kat}$ & $. / 9 .+4$ & $\mid \Psi / \cdot \mu^{D}$ & $\mid r / \cdot V^{D}$ & $|\omega /| \mathrm{f}^{\mathrm{ad}}$ & $\mid \omega / \Delta v^{a}$ & ماده خشك (درصد) \\
\hline.$/$ ter & g/Qf & $\| v r^{\mathrm{D}}$ & $\| V \varepsilon^{\mathrm{D}}$ & $1 r \Delta \cdot{ }^{\mathrm{a}}$ & $1 r q v^{a}$ & ماده خشك توليدى (كرم) \\
\hline \multicolumn{7}{|c|}{ عملكرد نسبى (كيلو كرم علف توليدى به ازاى كيلو كرم بذر مصرفى) } \\
\hline . /Tavı & . TEt & Q/TT & $\Delta / 1 \Lambda$ & r/Ar & f/vq & علف تازه به بذر مصرفى (بذر معمولى) \\
\hline$\cdot / \mu \cdot \Lambda$ & $\cdot /$ TAT & $\Delta / \wedge$. & $\Delta / \Psi \varphi$ & $\Delta / v \varepsilon$ & Q/Tr & علف تازه به بذر مصرفى (ماده خشك) \\
\hline.$/ 4 \& 4 V$ &.$/ N Q F$ & •/VQ & $\cdot / \mathrm{V} \Delta$ & $\cdot / \Lambda$. & $\cdot|\wedge|$ & نه بذر مصرفى (مليده خشك) (ماده خشك) \\
\hline \multicolumn{7}{|c|}{ توازن توليد در هر سينى (كرم ماده خشك توليدى منهاى گرم ماده خشك مصرفى از طريق بذر) } \\
\hline $.1 \cdot 1 \cdot r$ & $. / 4+\Lambda$ & $W / V \in v^{a}$ & $\mid Q / \Lambda r^{D}$ & $1 Q / M \cdot^{0}$ & $\mid w / q r \cdot \cdot^{c}$ & بخش سبزينه كياه (سانتىمتر) \\
\hline
\end{tabular}

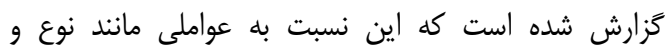

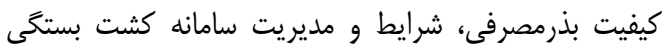

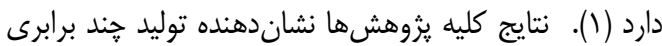

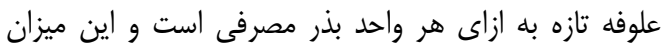

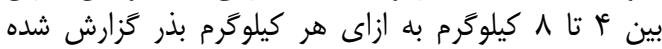

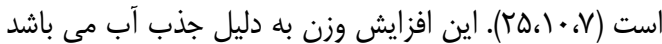

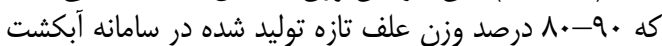

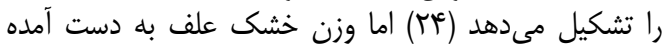

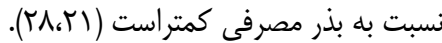

روند اتلاف ماده خشك و محتوى مواد مغذى دانهها طيى

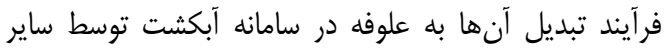

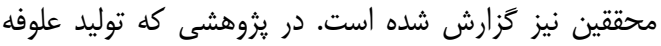

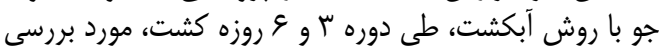

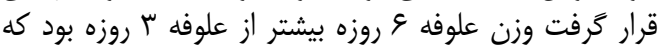
نشاندهنده افزايش جذب عاري روزي در طول دوره رشد است

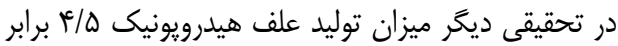

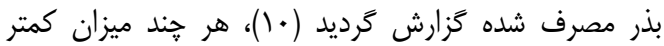
نيز (ن/VG) 
جدول r- تركيب شيميايى دانه جو، دانه ذرت و علوفه هيدرويونيك در روزهاى مختلف برداشت (كرم در صد گرم ماده خشك) Tabel 2. Chemical composition of barley grain, corn grain and hydropoinic greed fodder at different harvesting days

\begin{tabular}{|c|c|c|c|c|c|c|c|c|c|c|}
\hline \multicolumn{3}{|c|}{ احتمال معنىارى } & \multirow{2}{*}{ ميانكتينهاردا } & \multicolumn{4}{|c|}{ زمان برداشت علوفه (روز) } & \multirow[b]{2}{*}{ دانه ذرت } & \multirow[b]{2}{*}{ دانه جو } & \multirow[b]{2}{*}{ تركيبات } \\
\hline با ذيمار & تيمار & تيمارها & & 1. & 9 & $\wedge$ & V & & & \\
\hline$\cdot / \cdot \cdot v^{c}$ & $\cdot / \cdot 11$ & $.1 \cdot F \mathrm{WV}$ & . IVAV & $\mid \Psi / \cdot 9$ & $10 / 1 \pi$ & $1 \% / q T$ & $\mid Q / \Delta F$ & $\Lambda \Delta / M$ & $9 \pi / \pi r$ & DM \\
\hline.$\cdot \cdot \lambda r$ & ${ }^{\prime} \cdot \wedge \Delta$ & $\cdot 1 \cdot v+\Lambda$ & $\% V \Delta$ & $9 \Delta / V \Delta$ & $१ \Delta / \wedge \Delta$ & $98 / .0$ & $98 / 19$ & $9 N / \widetilde{q}$ & १४/q) & $\mathrm{OM}$ \\
\hline.$/ . .11$ &.$/ .91$ &.$/ 1 \vee 9 q$ &.$/ 1 v q$ & $\Delta / \wedge \Delta$ & $\varphi / 98$ & $r / \Lambda 1$ & $F / r$ & $9 / \cdot 1$ & 1/94 & $\mathrm{EE}$ \\
\hline$\cdot(\cdot \cdot v 1$ & $\%$. vg & $\cdot / \cdot r M$ & 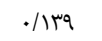 & $|\varepsilon / \mu|$ & $\mid Q / \Delta f$ & $1 \% / 98$ & $16 / \pi q$ & $11 / \pi r$ & $11 / 49$ & $\mathrm{CP}$ \\
\hline.$\cdot \cdot v r$ & 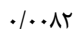 & $\cdot / 9 \ldots$ & $\cdot / 9$. & $F T / V I$ & $\varphi 1 / r$. & $r V / \& \Delta$ & $M F / F$ & $q / 4 q$ & $r \cdot 1 \Delta \Delta$ & NDF \\
\hline.$/ . v r$ & $\%$ vq & ./IVVe &.$/ \mathrm{vA}$ & $1 f / \pi q$ & r & $\mid r / 94$ & $11 / 99$ & $r / w r$ & $\mathrm{~V} / 11$ & $\mathrm{ADF}$ \\
\hline$\cdot|\cdot v|$ & $\cdot / \cdot \mathrm{v} \wedge$ & . ITrA & 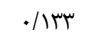 & $9 / \cdot V$ & $\Delta / \mathrm{V} \Lambda$ & $9 / .5$ & g/re & T/AT & $r / \Delta r$ & WSC \\
\hline.. $.9 \mu$ & • & $.1 \cdot 49 V$ & $. / a+V$ & ri/lf & $r F / \cdot \Delta$ & r & $r q / r$ & $V \backslash / ז q$ & $94 / \cdot 1$ & $\mathrm{NFC}$ \\
\hline $.1 . .90$ & .1 .99 & פ & 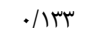 & $r / \Lambda S$ & $r / \Delta \Lambda$ & אس/r & $1 / 98$ & $1 / \cdot 9$ & $1 / 48$ & NDICP \\
\hline$\% \Delta Q q$ & $\%$. vq & $.1 .9 \mathrm{VV}$ & .1 .91 & $1 / 41$ & $r / T)$ & I/Tr & $1 / \pi r$ & $\cdot / \Delta \Delta$ & $\cdot 1 \Delta \theta$ & ADICP \\
\hline
\end{tabular}

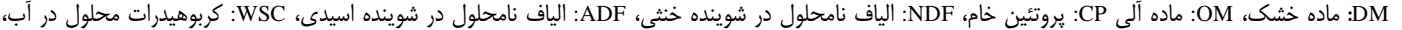

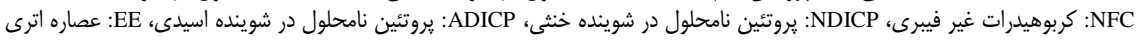

بروتئين خام مىشود كه البته اين افزايش از نظر مقدار

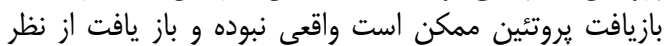

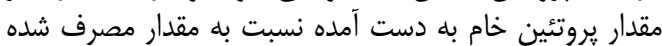

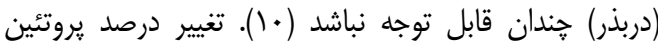

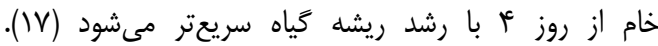

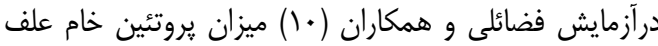

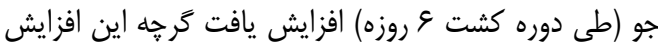

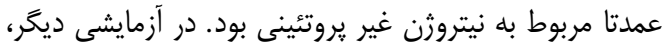

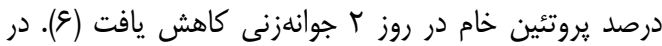

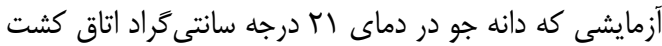

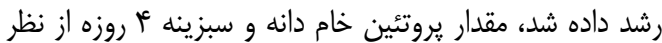

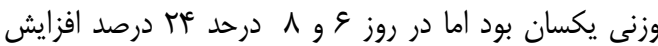

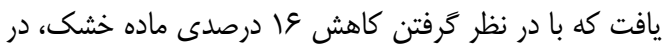

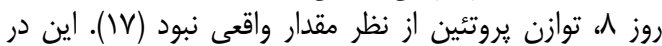

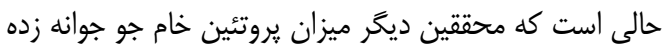

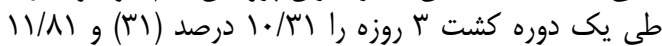

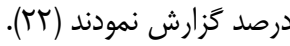
در صورت افزودن كودهاى نيتروزن دار، درصد برون بروتئين

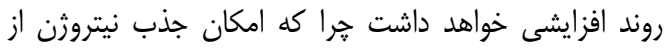

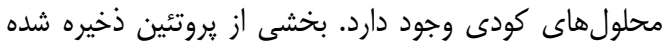

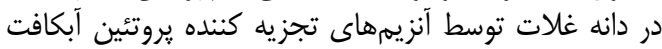

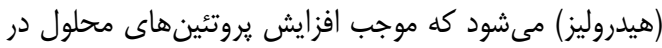

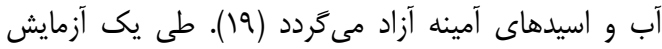

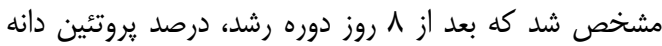

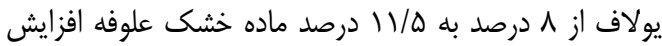

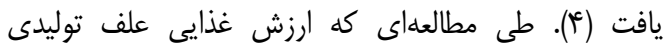

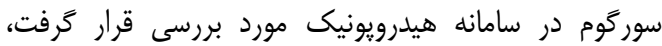

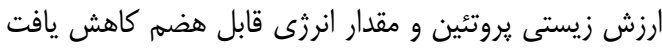

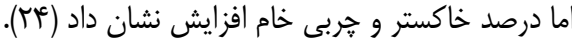

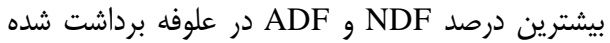

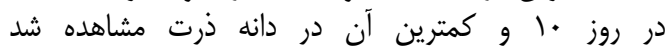

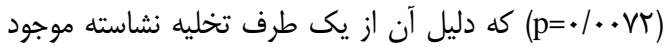

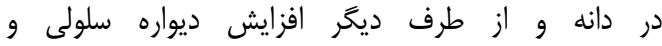

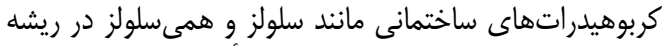
و برى مىباشد. شرايط رشد و نوع بذر تأثير بسزايى در تركيب
طى جوانهزنى و رشد كياه، فرآيندهاى متابوليكى فعال

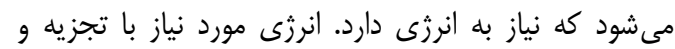

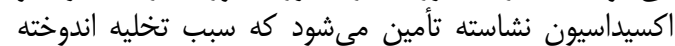

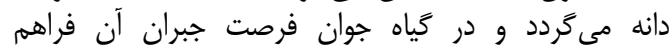

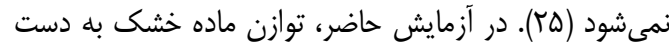

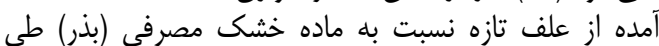

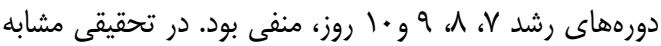

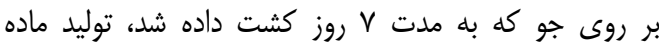

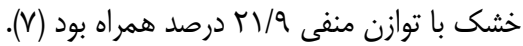
تركيب شيميايى همان طورى كه در جدول r ن نشان داده شده است، بـه جـز

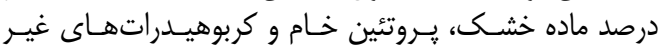

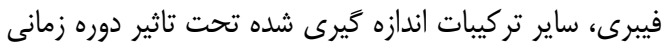

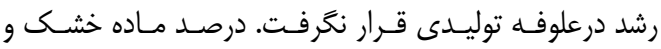

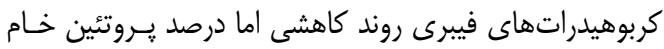

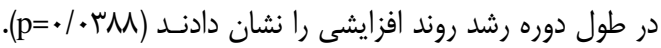

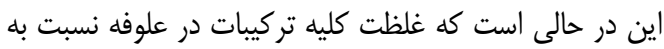

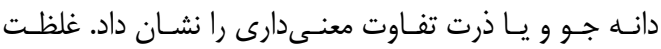

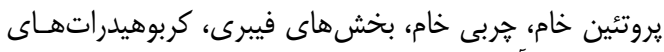

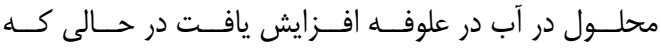

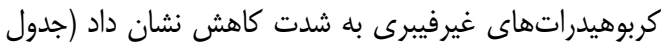

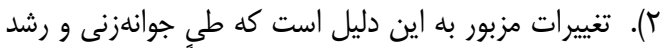

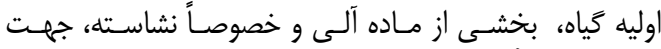

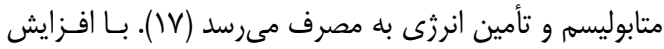

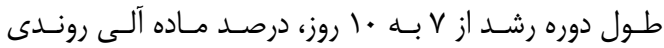

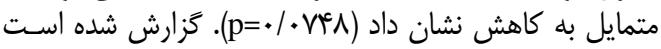

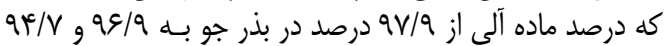

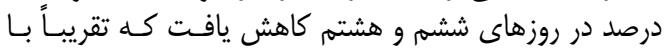
يافتهاى اين تحقيق مشابه است (IV)

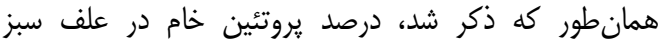

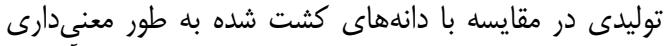

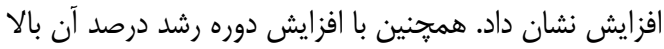

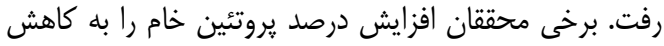

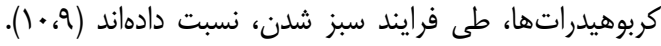
افزايش دوره رشد سبب كاهش بيشتر ماده خشك و افزيت دايش 


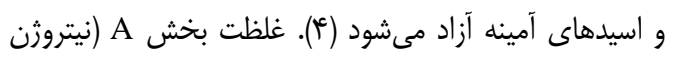

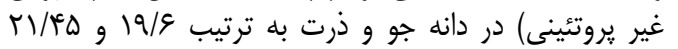

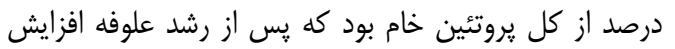

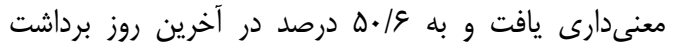

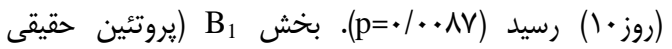

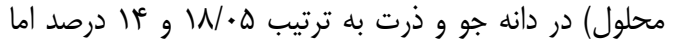

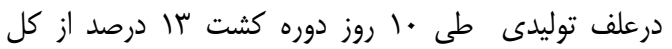

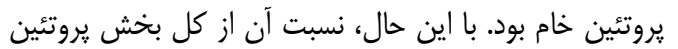

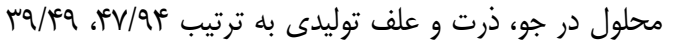

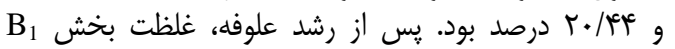

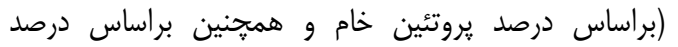
يروتئين محلول) كاهش يافت. بخش ماتش

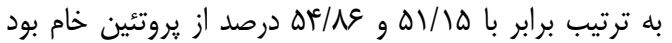

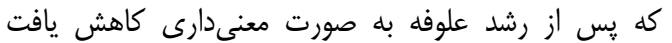

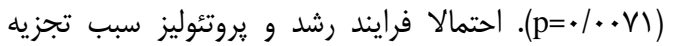
بخش قابل توجهى از B

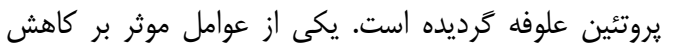

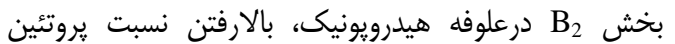

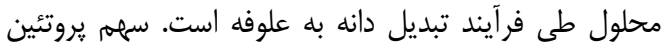

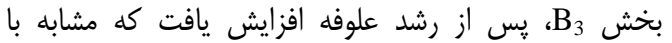

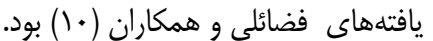

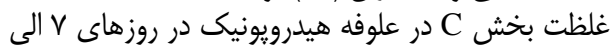

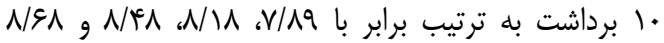

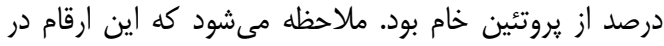

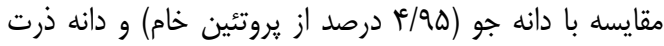

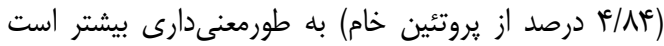

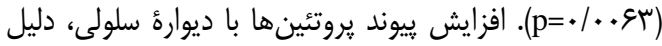

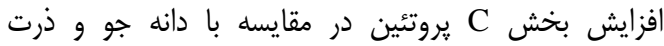

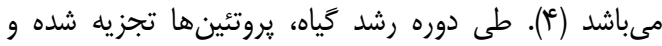

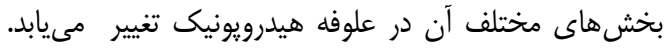

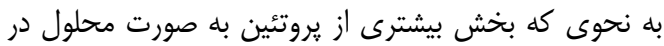

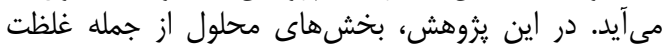

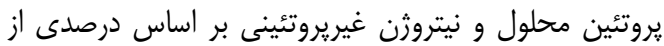

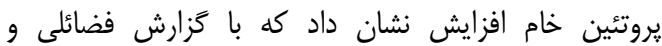

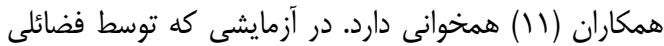

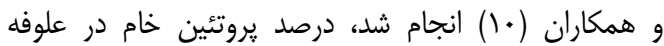

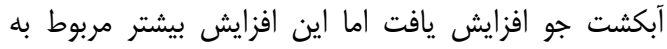

$$
\text { نيتروثن غيريروتئينى بود. }
$$

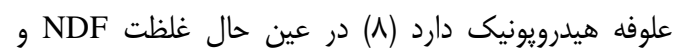

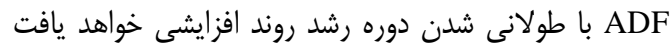

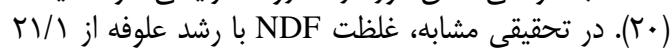

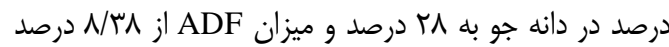

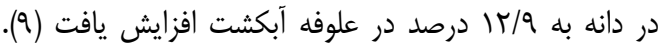

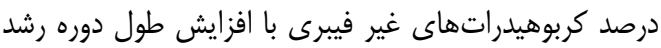

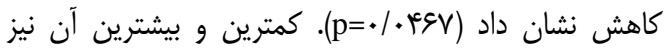

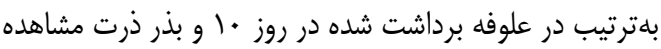

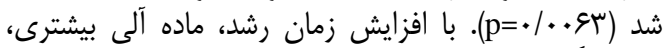

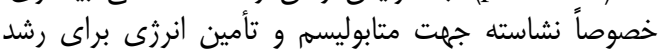

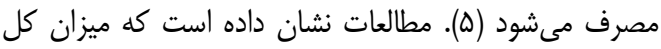

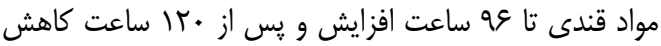

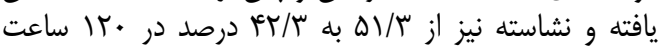

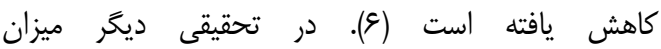

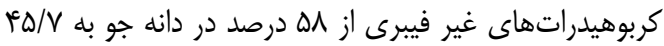

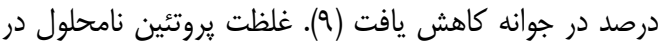

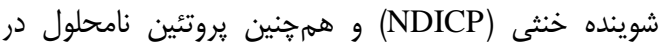

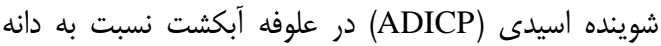

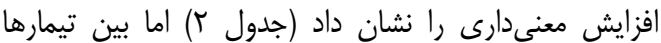

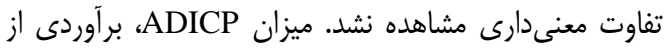

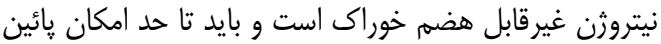

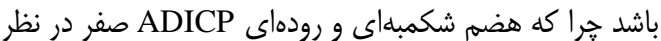

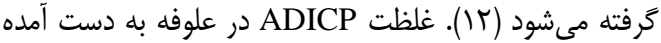

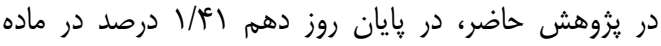

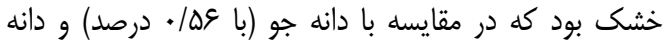

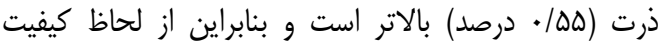

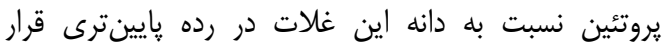

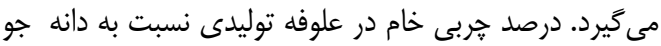

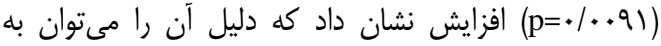

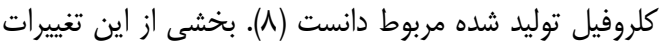

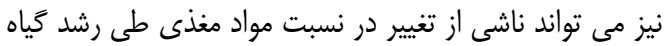

كيفيت يروتئين

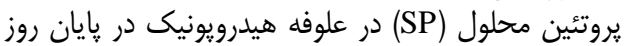

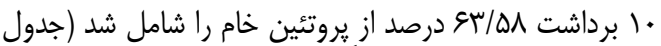

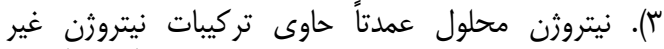

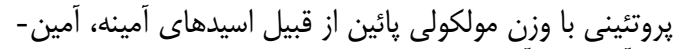

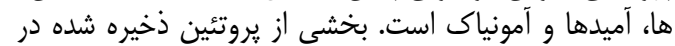

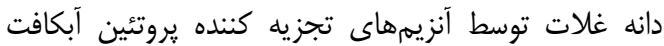

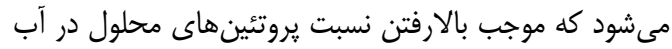


جدول זـ غلظت بخشهاى مختلف يروتئين (بر اساس روش CNCPS) در دانههاى جو و ذرت و علوفه هيدرويونيك Tabel 3. Protein fractions (based on CNCPS) in barley grain, corn grain and hydroponic green fodder

\begin{tabular}{|c|c|c|c|c|c|c|c|c|}
\hline $\mathrm{C}$ & $\mathrm{B}_{3}$ & $\mathrm{~B}_{2}$ & \multicolumn{2}{|c|}{$\mathrm{B}_{1}$} & \multicolumn{2}{|c|}{ A } & SP & \multirow{2}{*}{ تيمار \# } \\
\hline 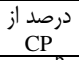 & $\begin{array}{l}\text { درصد از } \\
\text { دP }\end{array}$ & 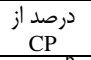 & $\begin{array}{c}\text { درصد از } \\
\text { SP }\end{array}$ & 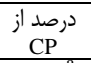 & درصد از & 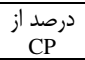 & 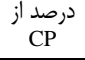 & \\
\hline$F / 9 \Delta^{\circ}$ & $9 / \pi \Delta^{\circ}$ & $\Delta \backslash / / Q^{\circ}$ & $F V / q q^{a}$ & $\mid N / \cdot D^{a}$ & $\Delta T / \cdot \Delta^{T}$ & $19 / 9.1$ & $r V / 9 Q^{\mathrm{a}}$ & دانه جو \\
\hline$F / \Lambda F^{D}$ & $r / \Lambda \Delta^{a}$ & $\Delta F / \mathcal{N}^{\mathrm{a}}$ & $r q /<q^{D}$ & $\mid F / .^{\mathrm{a}}$ & $8 \cdot|\Delta|^{e}$ & $r \mid / F \Delta^{e}$ & $\Gamma \Delta / \mathcal{F} \Delta^{\mathrm{e}}$ & علوفه: \\
\hline$V / \wedge q^{a}$ & $\Delta / Q Y^{c}$ & $r q / 19^{c}$ & $r q / \wedge r^{c}$ & $\mathrm{~V} / \mathrm{.}^{\mathrm{D}}$ & $v \cdot / / v^{a}$ & $f \cdot 1 . a^{a}$ & $\Delta \mathrm{V} / .^{\mathrm{c}}$ & jg $V$ \\
\hline$N / \Lambda^{a}$ & $V / F Y^{D}$ & $r \mu / \kappa^{a}$ & $r \Psi^{\prime} / q \Psi^{\mathrm{a}}$ & $10 / r^{c}$ & $V \Delta / \cdot \Lambda^{\mathrm{c}}$ & $F \Delta / \lambda .^{c}$ & $91 / .0^{0}$ & ^ روز \\
\hline$\Lambda / F \Lambda^{\mathrm{a}}$ & $N / 1 Q^{\mathrm{ab}}$ & $r \cdot / r r^{e}$ & $r \mu / l^{e}$ & $\mid F / F q^{c a}$ & $v \varepsilon / q^{D}$ & $F N / \Delta \cdot{ }^{D}$ & $q \mu / . .^{a}$ & 9 \\
\hline$N / 9 \Lambda^{a}$ & $N / \Lambda \Delta^{\mathrm{a}}$ & $\mathrm{N} / \wedge V^{\mathrm{I}}$ & $r \cdot / \kappa F^{I}$ & $1 \% / .^{e}$ & $\vee \vee / \Delta \gamma^{\mathrm{a}}$ & $0 \cdot / \varphi^{a}$ & $G H / \Delta \Lambda^{a}$ & . 1 روز \\
\hline.$/$ TVE & $\cdot /$ TVG &.$/ T V E$ & . & . & $\cdot / T \Delta S$ & $\cdot / \Delta T Y$ & 每/ & اشتباه استاندارد \\
\hline (1.. & $\cdot / \cdot \wedge \mu$ & $\cdot / \cdot v 1$ & $\cdot / \cdot \bullet 9 \Lambda$ & $\cdot / \cdot v^{e}$ &.. $.9 D$ & $\cdot / \cdot \wedge \mathrm{V}$ &.$/ .991$ & احتمال معنىدارى \\
\hline
\end{tabular}

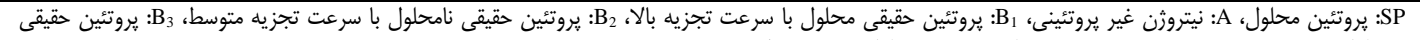

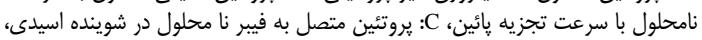

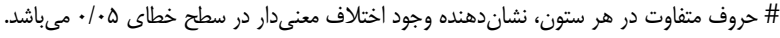

بود. اما طول دوره كشت اثرى برمتغير هاى مزبور در علوفه

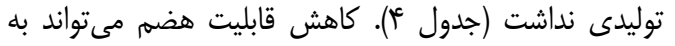

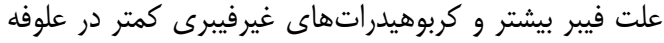
نسبت به دانه باشد.
مطابق جدول ؟َ، قابليت هضم ماده آلى علوفه توليدى،

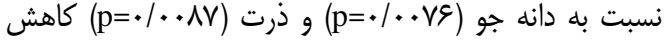

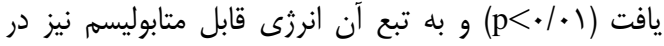

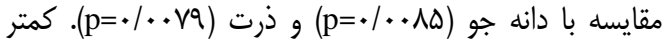

Tabel 4. DM and OM digestibility of grains and the green fodder

\begin{tabular}{|c|c|c|c|c|c|c|c|c|c|c|}
\hline \multicolumn{3}{|c|}{ احتمال معنى دارى } & \multirow{2}{*}{ استاندارد } & \multicolumn{4}{|c|}{ زمان برداشت علوفه (روز) } & \multicolumn{2}{|l|}{ دانه } & \multirow[b]{2}{*}{ فراسنجه } \\
\hline با ذرتمار & تيا جو & تيمارها & & 1. & 9 & 1 & V & ذرت & جو & \\
\hline$\cdot / \cdots \wedge \mathrm{V}$ & $\cdot 1+\cdot v 9$ & $\cdot / V \wedge \& \Lambda$ & $\cdot|\Delta \Delta|$ & $\wedge \backslash / ৭ \Lambda^{0}$ & $\Lambda \Gamma / \Delta q^{D}$ & $\Lambda \mu / \Psi^{D}$ & $\Lambda \Psi / \Psi \mathcal{F}^{D}$ & $9 \% / V V^{a}$ & $9 r / / f^{a}$ & OMD \\
\hline$\%$. vq & $\% \cdots \wedge \Delta$ & $\cdot \mid \cdot v^{m}$ & gr/. & $r / \Lambda \Gamma^{D}$ & $r / \wedge V^{D}$ & $r / \Lambda q^{D}$ & $r / q)^{D}$ & $r / \mu \cdot{ }^{a}$ & $\mu / \mid q^{a}$ & $\mathrm{ME}$ \\
\hline
\end{tabular}

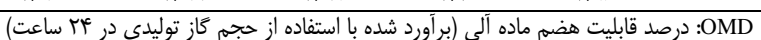

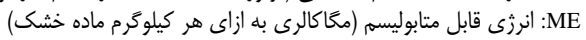

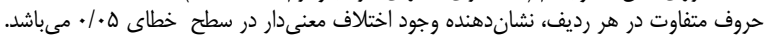

\section{بازده تغذيهاى}

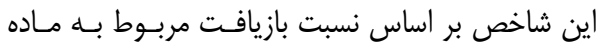

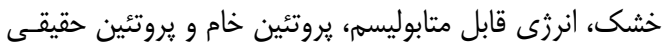

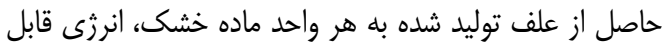

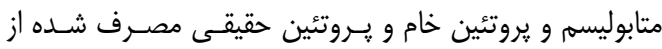

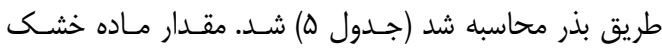

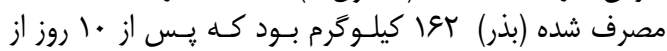

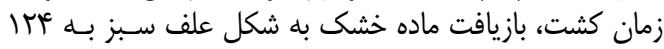

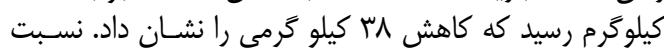

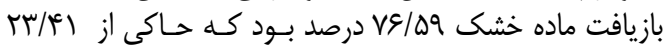

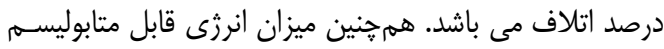

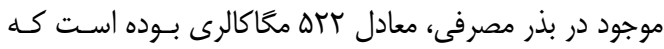

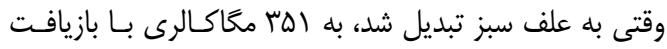

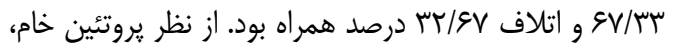

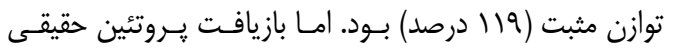

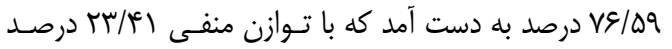

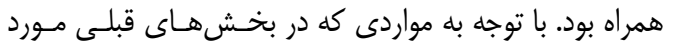

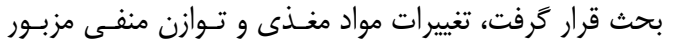
قابل توجيه مى باشد.
كاهش قابليت هضم ماده خشك و انرزى قابل

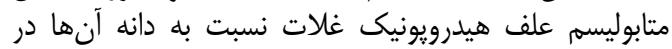

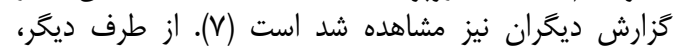

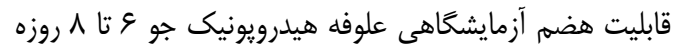

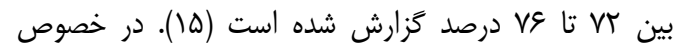

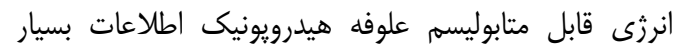

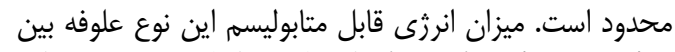

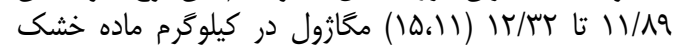

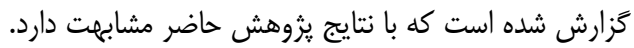

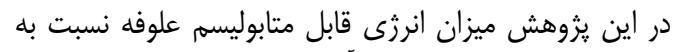

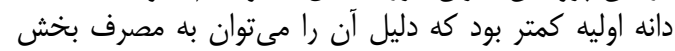

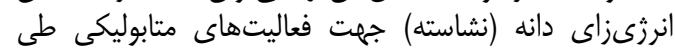

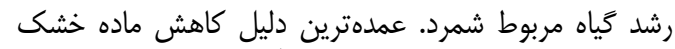

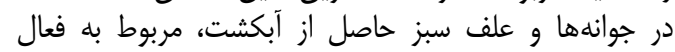

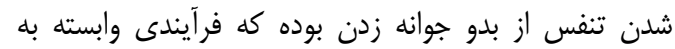

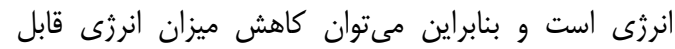

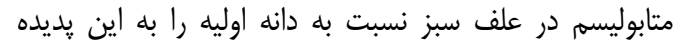

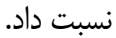

بازده سامانه 
جدول ه- بازده علوفه توليدى از نظر انرزى و مواد مغذى طى دوره كشت • ا روزه Table 5. Eficiency of green fodder for energy and nutrients, at 10 days growing period

\begin{tabular}{|c|c|c|c|c|}
\hline يروتئين حقيقى & يروتئين خام & انرزى قابل متابوليسم & ماده خشك & متغير \\
\hline $1 r^{a}$ & $1 \mathrm{~V}^{\circ}$ & $\Delta T r^{\mathrm{a}}$ & $19 \mathrm{r}^{\mathrm{a}}$ & مقدار مصرف شده (از طريق بذر)' \\
\hline $1.0^{0}$ & $r \cdot / r^{\mathrm{a}}$ & $r \Delta\rangle^{\mathrm{D}}$ & $1 \pi f^{0}$ & مقدار به دست آمده (علوفه توليدى)' \\
\hline$V \& / 0 q$ & 119 & שr/ & $V \& / 0 q$ & نسبت مقداربه دست آمده به مقدار مصرف شده (٪) \\
\hline.$/ 4)$ & . lat & .1 .48 & 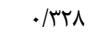 & اشتباه استاندارد ميانگينها \\
\hline $.1 . .94$ & $\cdot / \cdot 11$ & $\cdot / \cdot \vee \wedge$ & $.1 . .91$ & احتمال معنىدارى \\
\hline
\end{tabular}

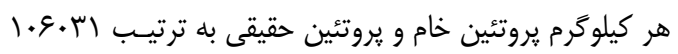

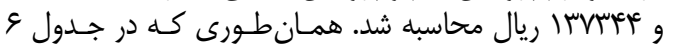

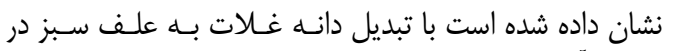

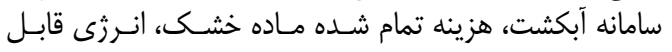

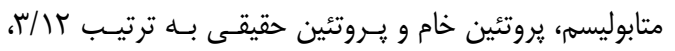

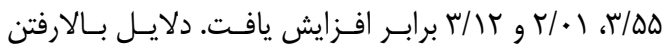

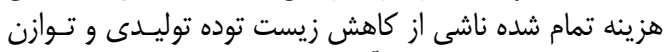

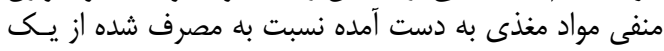

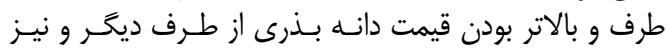

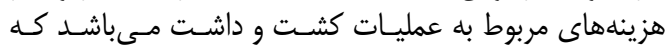

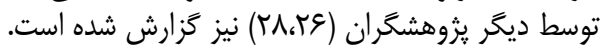

بازده از نظر هزينه

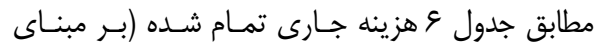

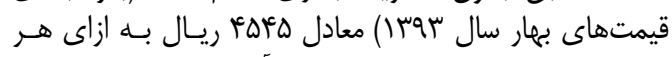

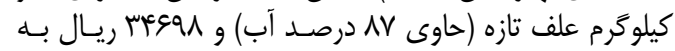

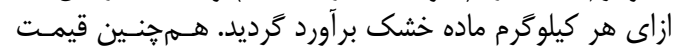

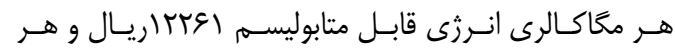
كيلو است كأ

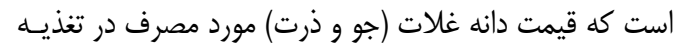

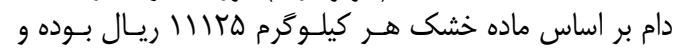

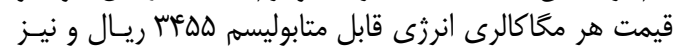

جدول צ - مقايسه هزينه تمام شده علف هيدرو يونيك و مواد مغذى محتوى آن نسبت به جو و ذرت Table 6. Comparing of nutrients cost in green fodder with barley and corn grains

\begin{tabular}{|c|c|c|c|}
\hline لنسبت هزينه \# & جو و ذرت & علف هيدرويونيك & شرح هزينه \\
\hline- & $1 \ldots \cdot$ & FAFD & برحسب As-fed (ريال در هر كيلو كرم) \\
\hline r/lr & $111 r \Delta$ & resqu & برحسب ماده خشك (ريال در هر كيلو گرم) \\
\hline$\Gamma / \Delta \Delta$ & TEDQ & $\mid$ ITYA & ريال به ازاى هر مخاكالرى انرزى قابل متابوليسم \\
\hline$r / \cdot 1$ & $1 \cdot 9 \cdot 41$ & TITAVT & ريال به ازاى هر كيلوكَرم بروتئين خام \\
\hline$r / 11$ & IrVmete & FTATRT & ريال به ازاى هر كيلوكرم يروتئين حقيقى \\
\hline
\end{tabular}

بالا رفتن هزينه خواهد شد. مشاهده آلودگى هاى قارجى در

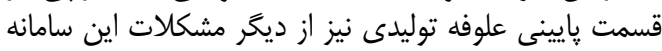

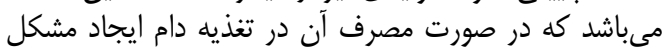

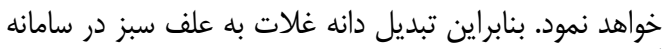

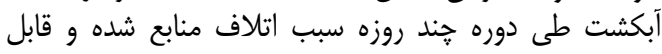
توصيه نمىباشد.

\section{تشكر و قدردانى}

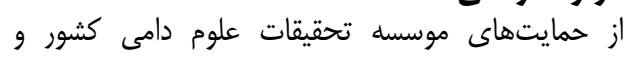

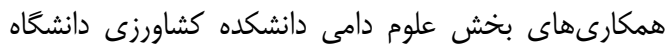

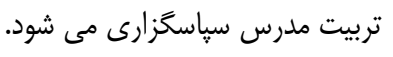

تبديل دانه غلات به حالت سبز شده در سامانه آبكشت،

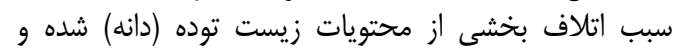

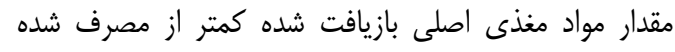

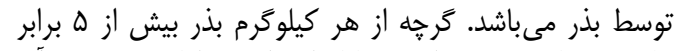

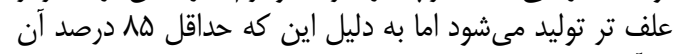

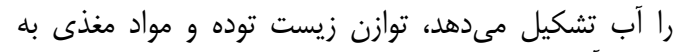

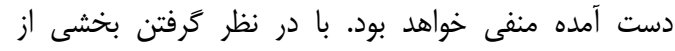
هزينههاى مصرفى، هر واحد علوفه توليدى امدي (برحسب ماده

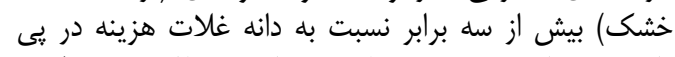

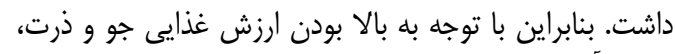

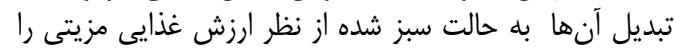

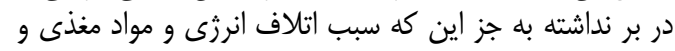


1. Al-Ajmi, A.A., I. Salih, I. Kadhim and Y Othman 2009. Yield and water use efficiency of barley fodder produced under hydroponic system in GCC countries using tertiary treated sewage effluents. Phytology, 1: 342-348.

2. Al-Karaki, N. Ghazi and N. Al-Momani. 2011. Evaluation of some barley cultivars for green fodder production and water use efficiency under hydroponic conditions. Jordan Journal of Agricultural Sciences, 7: 448-457.

3. AOAC. 1990. Official methods of analysis, $15^{\text {th }}$ Edition. Association of Official Analytical Chemists, Washington, DC, USA.

4. Chavan, J. and S.S. Kadam. 1989. Nutritional improvement of cereals by sprouting. Critical Reviews in Food Science and Nutrition, 28: 401-437.

5. Chung, T.Y., E.N. Nwokolo and J.S. Sim. 1989. Compositional and digestibility changes in sprouted barley and canola seeds. Plant Foods in Human Nutrition, 39: 267-278.

6. Dagnia, S., D. Petterson, R. Bell and F. Flanagan. 1992. Germination alters the chemical composition and protein quality of lupin seed. Journal of the Science, Food and Agriculture, 60: 419-423.

7. Dung, D.D., I.R. Godwin and J.V. Nolan. 2010. Nutrient content and in sacco digestibility of barley grain and sprouted barley. Journal of Animal and Veterinary Advances, 9: 2485-2492.

8. Fayed, A.M. 2011. Comparative study and feed evaluation of sprouted barley grains on rice straw versus tamarix mannifera on performance of growing barki lambs in sinai. Journal of American Science, 7: 954961.

9. Fazaeli, H., H.A. Golmohammadi, A.A. Shoayee, N. Montajebi and S.H. Mosharraf. 2011. Performance of feedlot calves fed hydroponics fodder barley. Journal of Agriculture Science and Technology, 13: 367-375.

10. Fazaeli, H., H.A. Golmohammadi, S.N. Tabatabayee and M. Asghari-Tabrizi. 2012. Productivity and nutritive value of barley green fodder yield in hydroponic system. World Applied Science, 16: 531-539.

11. Fazaeli, H. 2014. Efficiency of hydroponic green fodder as animal feed. Animal Science Journal (Pajouhesh \& Sazandegi), 103: 205-214 (In Persian).

12. Fox, D.G., T.P. Tylotki, L.O. Tedeschi, M.E. Van Amburgh, L.E. Chase, A.N. Pell, T.R. Overton and J.B Russel. 2003. The net carbohydrate and protein system for evaluating herd nutrition and nutrient excretion: CNCPS, Version 5.0. Department of Animal Science, Cornell University, Ithaca, New York, 294 pp.

13. Greenberg, N.A. and W.P. Shipe. 1979. Comparison of the abilities of trichloroacetic, picric, sulfosalicylic, and tungestic acids to precipitate protein hydrolysates and proteins. Journal of Food Science, 44: 735-737.

14. Licitra, G., T.M. Hernandez and P.J. Van Soest. 1996. Standadization of procedures for nitrogen fractionation of ruminant feeds. Animal Feed Science and Technology, 57: 347-358.

15. Mansbridge, R.J. and B.J. Gooch. 1985. A nutritional assessment of hydroponically grown barley for ruminants. Animal Production, 4: 569-570.

16. Menke, K.H. and Y.H. Stingass. 1988. Estimation of the energetic feed value obtained from chemical analysis and in vitro gas production using rumen fluid. Animal Research and Developments, 28: 7-55.

17. Morgan, J., R.R. Hunter and R.O'. Haire. 1992. Limiting factors in hydroponic barlev grass production. Proceeding of the $8^{\text {th }}$ international congress on soil less culture, 241-261 pp. Hunter's Rest, South Africa.

18. NRC. 2001. Nutrient reqirements for dairy cattle. Academy Press, Washington, DC. 360 pp.

19. Nielson, M.T., R.E. Meade, G.M. Paulsen and R.C. Hoseney. 1978. Improvement of wheat protein quality by germination. Proceedings of the $10^{\text {th }}$ national conference on wheat utilization research, 23-39 pp., Tucson, Arizona, USA.

20. Pandey, H.N. and N.N. Pathak. 1991. Nutritional evaluation of artificially grown barley fodder in lactating crossbred cows. Indian Journal of Animal Nutrition, 8: 77-78.

21. Peer, D.J. and S. Leeson. 1985. Feeding value of hydroponically produced barley for poultry and pigs.Animal Feed Science and Technology, 13: 183-190.

22. Rasteh, M.R. and B. Dastar. 2015. Determination of chemical composition and metabolisable energy of germinated barley in broiler chickens. Research on Animal Production, 6: 1-8 (In Persian).

23. SAS, Statistical Aanalysis System. 2001. Users Guide, Statistics, version 8.2. SAS Institute, Inc., Carry, NC.

24. Shem, M., F. Lekule, G. Zakayo and B. Eggum. 1990. Nutritive value of germinated and un-germinated high tannin sorghum for growing pig. Acta Agriculture Scandinavica, 40: 253-258.

25. Sneath, R. and F. McIntash. 2003. Review of hydroponic fodder production for beef cattle. Department of primary industries. Queenlands Australia. McKeehen. 55 pp.

26. Thomas T.A. 1977. An automated procedure for the determination of soluble carbohydrate in herbage. Journal of Science of Food and Agriculture, 28: 639-642.

27. Tranel, L.F. 2013. Hydroponic fodder systems for dairy cattle. Iowa, ISU Extension and Outreach, A.S. Leaflet R2791. at: lib.dr.iastate.edu/ans air/vol659/iss1/42

28. Trubey, C.R. and Y. Otros. 1969. Effect of light, culture solution and growth period on growth and chemical composition of hydroponically produced oat seedlings. Agronomy, 61: 663-665.

29. Tudor, G., T. Darcy, P. Smith and F. Shallcross. 2003. The intake and liveweight change of droughmaster steers fed hydroponically grown, young sprouted barley fodder (autpgrass). In: Review of hydroponic fodder production for beef cattle, Project Report. Western Australia. $55 \mathrm{pp}$.

30. Van Soest, P.J., J.B. Robertson and B.A. Lewis. 1991. Methods for dietary fiber, neutral detergent fiber and nonstarch polysaccharides in relation to animal nutrition. Journal of Dairy Science, 74: 3583-3597.

31. Veysi, A., A. Afzalzadeh, H. Fazaeli and H. Baneh. 2015. Determination of chemical composition, digestibility and dry matter and protein degradability parameters of three-days sprouted barley. Research on Animal Production, 6: 115-122 (In Persian). 


\title{
Nutritive Value and Performance of Cereal Green Fodder Yield in Hydroponic System
}

\author{
Hassan Fazaeli ${ }^{1}$, Somayeh Solaymani ${ }^{2}$ and Yousef Rouzbahan ${ }^{3}$ \\ 1- Professor, of Animal Science Research Institute, The Research, Education and Extension, Karaj, Iran \\ (Corresponding author: h hfazaeli@gmail.com ) \\ 2 and 3- Graduate M.Sc. Student and Associate Professor, Faculty of Agriculture, Tarbiat Modarres University, \\ Tehran \\ Received: December 22, $2014 \quad$ Accepted: August 25, 2015
}

\begin{abstract}
This study was conducted to assess the nutritive value and performance of green fodder (GF) production in hydroponic system. A mixture of barley $(100 \mathrm{~kg})$ and corn $(80 \mathrm{~kg})$ seeds was prepared and grown in an automatic controlling growing chamber that contained 7 shelevs, each with 16 special trays capacity. From each shelf, 6 trays were taken out after 7, 8, 9 and 10 days (as treatment) and fresh weight was recorded and sampled. Chemical composition and in vitro digestibility was determined. The performance and cost of GF production were estimated. Results showed that the fresh GF yield was 5.25 times of the original seed nevertheless, dry matter obtained was $23.8 \%$ less than the initial spent seed. Crude protein (CP) content of GF was significantly higher than that of barley $(P=0.0076)$ and corn grain $(P=0.0071)$ but such elevation in $\mathrm{CP}$ was mainly related to non-protein nitrogen. Organic matter digestibility of GF reduced as compared to the barley $(P=0.0079)$ and corn grain $(P=0.0087)$. The ME content was reduced in green fodder when compared to barley grain $(P=0.0085)$ and corn grain $(P=0.0079)$. No differences were obtained among the GF treatments for nutrient contents and igestibility.The estimated cost per kg DM, CP, TP and ME obtained from GF were respectively 3.12, 2.01, 3.12 and 3.11 times of barley and corn grains. Ovreall, not only there is a negative balance of utrients during converting barley and corn grains to green fodder in hydroponic system, but also the rice of nutrients obtained by GF is several times higher than the original grains.
\end{abstract}

Keyword: Barley, Corn, Efficiency, Green Fodder, Hydroponic 